\title{
Absolute myocardial blood flow quantification with SPECT/CT: Is it possible?
}

\author{
Piotr J. Slomka, PhD, ${ }^{\mathrm{a}}$ Daniel S. Berman, $M D,{ }^{\mathrm{b}, \mathrm{c}}$ and Guido Germano, $\mathrm{PhD}^{\mathrm{b}, \mathrm{c}}$ \\ a Department of Medicine, Cedars-Sinai Medical Center, Los Angeles, CA \\ ${ }^{b}$ Departments of Imaging and Medicine, and Cedars-Sinai Heart Institute, Cedars-Sinai Medical \\ Center, Los Angeles, CA \\ c David Geffen School of Medicine, University of California Los Angeles, Los Angeles, CA
}

doi: $10.1007 / \mathrm{s} 12350-014-0002-6$

\section{VALUE OF ABSOLUTE FLOW}

Absolute myocardial blood flow (MBF) and myocardial flow reserve (MFR) are important physiological variables related to heart disease. Traditionally, these measurements have been obtained by dynamic PET imaging. They have been shown to provide clinically useful information beyond that provided by the assessment of relative perfusion defects in a variety of ways. These variables can potentially identify multivessel coronary artery disease (CAD) and predict the extent of disease more accurately than relative quantification or visual analysis of myocardial perfusion imaging (MPI) ${ }^{1,2}$ which are the clinical standards today. The ability to quantify absolute MBF with stress MPI may permit improved identification of patients in whom the relative regional distribution of tracer may appear normal because of a balanced reduction of blood flow. ${ }^{3}$ Prognostically, MFR demonstrated value as a marker for adverse cardiac events, allowing additional risk stratification beyond that provided by the assessment of relative perfusion defects alone. ${ }^{4-7}$ Even in the absence of obstructive epicardial disease, a low value of stress MBF or MFR can predict increased risk of cardiac events, and therefore, indicate the possible need of aggressive therapy for possible risk factors of CAD. ${ }^{8}$

\section{WHY IS FLOW QUANTIFICATION NOT WIDELY USED?}

Despite promising clinical value of MBF and MFR, these variables are still rarely used in the standard

\section{See related article, pp. 1075-1088}

\footnotetext{
Reprint requests: Piotr J. Slomka, Department of Medicine, Cedars-

Sinai Medical Center, Los Angeles, CA; slomkap@cshs.org

J Nucl Cardiol 2014;21:1092-5.

$1071-3581 / \$ 34.00$

Copyright (C) 2014 American Society of Nuclear Cardiology.
}

clinical practice today. Why? Currently, PET MPI imaging in the United States constitutes a small fraction $(<5 \%)$ of all MPI due to the relatively expensive equipment as compared to SPECT, limited availability of $\mathrm{Rb}-82$ generators, and few cardiac centres with available cyclotrons. Thus, SPECT is by far the most popular technique, with over 7 million scans performed in the U.S. annually. ${ }^{9}$ SPECT MPI is, however, limited to visual analysis or, at most, relative perfusion quantification. ${ }^{10}$ Even if PET MPI is performed, flow quantification is not always obtained, since it may require the latest PET/CT equipment with high count rate capability and specialized software. Furthermore, reduced MFR and MBF could represent subclinical microvascular disease and thus represent false positive finding with respect to obstructive disease-currently, however, the modification of patient treatment in such scenarios is complex and yet standardized. Nevertheless, if it were possible to perform flow quantification with SPECT as a part of standard SPECT MPI scans it would open up the possibility of much wider clinical evaluation and application of this technique than is currently possible with PET.

\section{ABSOLUTE FLOW QUANTIFICATION WITH CONVENTIONAL TC-99M SPECT}

In this issue of Journal of Nuclear Cardiology ${ }^{\circledR}$, Klein et al describe pilot results from dynamic SPECT/ CT imaging in 28 patients obtained in Taiwan on a conventional Anger camera (Siemens Symbia SPECT/ CT) with a standard parallel hole collimator. ${ }^{11}$ Several noteworthy technical points should be highlighted in regard to this study. The dual head SPECT scanner utilized does allow fast $90^{\circ}$ rotations (10 seconds), thus allowing acquisition of 10 -second SPECT frames. The dose for stress $(10 \mathrm{mCi})$ was relatively low (stress-first 
protocol). Latest iterative reconstruction with CT attenuation correction, scatter correction, and resolution recovery has been used, and phantom calibration was performed to obtain true quantitative $\mathrm{Bq} / \mathrm{cm}^{3}$ tracer concentrations. The flow quantification algorithm was based on their commercially available quantitation package designed for PET MPI analysis (FlowQuant), which uses a 1-tissue compartment model, with fixed ratio of $\mathrm{K} 1$ (uptake) to $\mathrm{k} 2$ (washout). The MIBI extraction fraction correction function assumed in the algorithm was based on the animal model developed originally by Leppo et $\mathrm{al}^{12}$ Authors reprocessed the data several times to evaluate inter and intra-operator variability for the MFR and MBF. Although there was no "gold standard" available in this study, (such as PET), they found a significant reduction of MBF and MFR in the high risk vs low risk population. They also compared standard relative perfusion measures (summed stress scores) to flow measurements and found good correlation between these variables. This is a novel study and is likely to stimulate discussion in the nuclear cardiology community regarding new possibilities of obtaining absolute flow measurements. While this is an exciting development, several key limitations must be considered.

\section{IS TC-99M MIBI TRACER GOOD ENOUGH?}

The Tc-99m MIBI is less than optimal for the measurement of the flow due to its relatively poor myocardial extraction fraction as compared to other SPECT tracers such as Tl-201. ${ }^{2}$ This is compensated for to some extent by a higher retention and less flow-dependent nature of this tracer, as compared to Tl-201. ${ }^{2}$ The Tc-99m MIBI extraction fraction is also much lower than the extraction fraction of PET tracers such as N-13 ammonia. ${ }^{12,13}$ Nevertheless, when the extraction fraction of Tc99m MIBI (Fig. 3 in Leppo et $\mathrm{al}^{12}$ ) is compared to that of Rb-82 (Fig. 9 in Yoshida et al. ${ }^{13}$ ), it is only slightly lower for Tc-99m MIBI for flows between 1 and $3 \mathrm{~mL} \cdot \mathrm{min}-$ $u^{-1} \cdot \mathrm{g}^{-1}$. To our knowledge, a direct Rb-82 to Tc-99m MIBI comparison was not performed in the same experiment. Given that these separate studies used different animal models (dogs for Rb-82 ${ }^{13}$ vs rabbits for Tc-99m $\mathrm{MIBI}^{12}$ ), the comparison above is approximate.

Although the Tc-99m is definitely not an optimal flow tracer, the competitive tracers have their own limitations. Tl-201 has less optimal imaging energy and is associated with higher radiation to the patient, limiting the injected dose. The use of N-13 and O-15 is confined to the sites with nearby cyclotrons. The SPECT image tracer, Tc99m-teboroxime with very high extraction fraction, approved for clinical use by the FDA over 20 years ago, has been proposed for examining absolute perfusion based on first-pass kinetics. ${ }^{14}$
However, Tc-99m teboroxime protocols are technically challenging due to high and rapid hepatic accumulation and very rapid washout of the tracer. The current closest competitor to Tc-99m MIBI in terms of practical use is $\mathrm{Rb}-82$, but it requires more expensive PET instrumentation and the availability of an onsite $\mathrm{Rb}-82$ generator. In the near future, we may see more developments in dynamic PET with the F-18 flurpiridaz tracer, ${ }^{15}$ which may be superior to Rb-82 due to higher extraction fraction and better image resolution, but it is not yet approved for clinical use. One key advantage of the Rb82 tracer is the possibility of back-to-back stress and rest imaging in one scanning session due to the 75 seconds half life of Rb-82. ${ }^{1}$ The Tc-99m protocol followed by Klein et al employed a 3-hour delay between stress and rest scans to reduce interference of the stress activity with rest scans. It also required delay of the acquisition for the static perfusion scans. Thus, the length of the full stress-rest Tc-99m perfusion protocol was over 5 hours, reducing its practical appeal. Nevertheless, despite these limitations, Tc-99m flow imaging with SPECT is certainly worth a careful look. If it were to become validated when compared to a standard such as one of the PET MPI approaches, it could turn out to be the most practical and cost-effective choice of all.

\section{IS CONVENTIONAL MPS SCANNER GOOD ENOUGH?}

Klein et al demonstrate the feasibility of the dynamic SPECT flow measurements with conventional dual head SPECT detectors. There are several major technical limitations which need to be pointed out in this approach. The temporal resolution of the kinetic frames is limited by the slow gantry rotation because of the time required to mechanically rotate the dual head detector around through the $90^{\circ}$ arc. Previously, a spatiotemporal iterative reconstruction methods to overcome these limitations had been investigated. ${ }^{16}$ Nevertheless, newer models of the conventional equipment as employed in this study allow fast back-and-forth rotation of 90 degrees with rotation times as short as 10 seconds. This is the timing resolution used in most $\mathrm{Rb}-82$ and PET studies, ${ }^{17,18}$ and therefore it should not limit practical clinical applications. More importantly, the conventional cameras have very poor count sensitivity, which is further amplified by the poor extraction fraction of Tc$99 \mathrm{~m}$. In a recent study by Verger et al, ${ }^{19}$ it has been demonstrated that the conventional dual head scanner with high-sensitivity parallel hole collimators detects less than $1 \mathrm{ppm}$ of the injected Tc-99m activity during the myocardial perfusion study. This is further exacerbated in the study by Klein et al by the use of stress-first low dose protocols $(10 \mathrm{mCi}$ stress dose $)$. Thus, the 
protocol described is truly testing the low count limitations, especially for the early 10-second frames, a period that is of critical importance in quantification of absolute flow for the definition of the input function. Despite such unfavorable count statistics, authors are able to achieve reasonable expert intra-observer reproducibility and reliably define the regions of interests for the myocardium and blood pool. However, it should be noted that the inter-observer reproducibility of the MFR was rather poor and may be related to the low count statistics apart from the experience of the observers. Another potential difficulty with conventional SPECT is a relatively poor spatial resolution in comparison to PET. This will affect the spillover between the blood pool and tissue. However, it is likely to be a less important effect than the count statistics, as the camera resolution is only partially responsible for the final image resolution, which is also affected by the cardiac, respiratory and patient motion. New resolution recovery techniques developed for SPECT, as used in the study by Klein et al may help in mitigating this effect.

\section{NEW OPPORTUNITY FOR HIGH-EFFICIENCY MPI}

As pointed out above, the primary limitation of dynamic imaging with conventional MPS scanners is the count sensitivity. High-efficiency MPI with innovative designs of the gantry, detectors, collimators, and image reconstruction has been recently developed to reduce imaging time and radiation dose. ${ }^{20}$ High-efficiency MPI can achieve simultaneous improvement in sensitivity (5-8 times higher) $)^{21}$ and image resolution (up to 2 times higher). Two vendors have introduced scanners with Cadmium-Zinc-Telluride detectors, coupled with highsensitivity collimation (multi-pinhole or high-sensitivity, parallel-hole-focusing on the myocardium). It is estimated that over 300 of these scanners are currently in use - a number that is increasing by about 100 per year ${ }^{22}$ and already surpassing the number of cardiac PET scanners. To date, these new techniques have been utilized primarily to reduce the routine scan time and radiation dose, ${ }^{23,24}$ or achieve ultra-low radiation dose $(<1 \mathrm{mSv})$ stress-only imaging ${ }^{25}$ at standard times (1012 minutes). The increased count sensitivity of these systems offers also a great advantage for the dynamic flow imaging. In Figure 1, we show comparative count sensitivity of the high-efficiency MPI, conventional MPI and PET, and demonstrate that the high-efficiency MPI count sensitivity, although still lower than 2D PET, is similar in magnitude. Most of the quantitative PET flow imaging was validated with 2D PET imaging. Initial studies of dynamic imaging have already been performed with the new high-efficiency SPECT systems. For instance, Ben-Haim et al studied 95 patients with

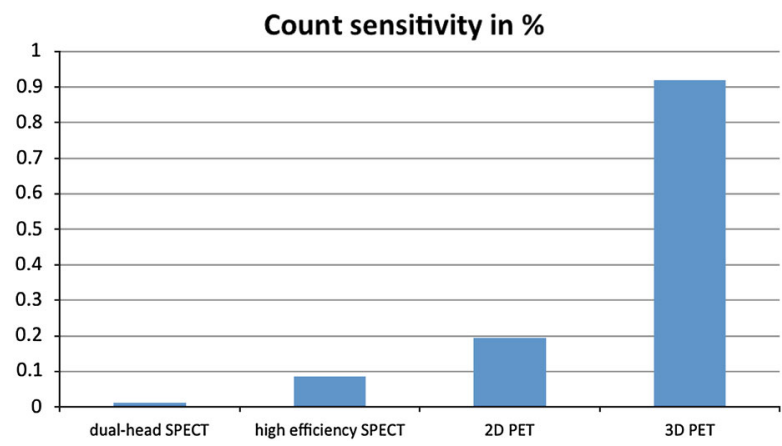

Figure 1. Relative count sensitivity of conventional dual head SPECT, high efficiency-SPECT, 2D PET and 3D PET. Based on Imbert et al, ${ }^{28}$ Mawlawi et $\mathrm{al}^{29}$.

high-efficiency MPI and reported correlation of MFR index with total perfusion deficit (TPD) relative quantification and obstructive angiographic findings in 20 patients. ${ }^{26}$ Wells et al have applied dynamic flow quantification analysis as described by Klein et al to animal images obtained with high-efficiency MPI, demonstrating very good correlations of MBF with invasive microsphere measurements. ${ }^{27}$

\section{FUTURE OUTLOOK AND CHALLENGES}

Enabling quantitative flow analysis with Tc-99m MPI would likely lead to much wider use of these key physiological variables in clinical practice. Several obstacles still need to be overcome to accomplish this goal. The low count statistics will remain a challenge for the conventional MPS. Fast-MPS imaging may solve this problem, but it is not yet widely used. In particular, the availability of CT-based attenuation correction is not common on these new systems. Extensive validation is needed, particularly by direct comparison to a gold standard, such as PET measurements with $\mathrm{N}-13$ or $\mathrm{O}-15$. The variability of the results due to the very low count statistics may need to be evaluated in test-retest studies. The long delay required between stress and rest Tc-99m imaging may limit practical applications; therefore, in addition to MFR, the value of stress-only peak flow (MBF) measurements with Tc-99m MIBI should be evaluated. Finally, clinical algorithms integrating relative perfusion measurements with absolute flow measurements and other clinical and imaging data need to be firmly establishedboth with the current PET data as well as with future SPECT assessments- to optimally guide patient management and demonstrate the added value of this new tool.

\section{Disclosures}

Dr. Berman is a shareholder in Spectrum Dynamics. Dr. Slomka has received a NIH grant related to cardiac SPECT 
(R01HL089765) and a Siemens research grant related to PET dynamic flow and quantitative PET analysis. Cedars-Sinai Medical Center receives royalties for the quantitative assessment of SPECT and PET, a portion of which is distributed to some of the authors (DB, GG, PS).

\section{References}

1. Hajjiri MM, Leavitt MB, Zheng H, Spooner AE, Fischman AJ, Gewirtz H. Comparison of positron emission tomography measurement of adenosine-stimulated absolute myocardial blood flow versus relative myocardial tracer content for physiological assessment of coronary artery stenosis severity and location. JACC Cardiovasc Imaging 2009;2:751-8.

2. Fiechter M, Ghadri JR, Gebhard C, Fuchs TA, Pazhenkottil AP, Nkoulou RN, et al. Diagnostic value of $13 \mathrm{~N}$-ammonia myocardial perfusion PET: Added value of myocardial flow reserve. J Nucl Med 2012;53:1230-4.

3. Ziadi MC, Dekemp RA, Williams K, Guo A, Renaud JM, Chow BJ, et al. Does quantification of myocardial flow reserve using rubidium82 positron emission tomography facilitate detection of multivessel coronary artery disease? J Nucl Cardiol 2012;19:670-80.

4. Herzog BA, Husmann L, Valenta I, Gaemperli O, Siegrist PT, Tay FM, et al. Long-term prognostic value of $13 \mathrm{~N}$-ammonia myocardial perfusion positron emission tomography added value of coronary flow reserve. J Am Coll Cardiol 2009;54:150-6.

5. Fukushima K, Javadi MS, Higuchi T, Lautamaki R, Merrill J, Nekolla SG, et al. Prediction of short-term cardiovascular events using quantification of global myocardial flow reserve in patients referred for clinical $82 \mathrm{Rb}$ PET perfusion imaging. J Nucl Med 2011;52:726-32.

6. Ziadi MC, Dekemp RA, Williams KA, Guo A, Chow BJ, Renaud $\mathrm{JM}$, et al. Impaired myocardial flow reserve on rubidium-82 positron emission tomography imaging predicts adverse outcomes in patients assessed for myocardial ischemia. J Am Coll Cardiol 2011;58:740-8.

7. Murthy VL, Naya M, Foster CR, Gaber M, Hainer J, Klein J, Dorbala $\mathrm{S}$, et al. Improved cardiac risk assessment with noninvasive measures of coronary flow reserve. Circulation 2011;124: 2215-24.

8. Schindler HS TH, Quercioli A, DIlsizian V. Cardiac PET imaging for the detection and monitoring of coronary artery disease and microvascular health. J Am Coll Cardiol 2010;3:623-40.

9. Einstein AJ. Effects of radiation exposure from cardiac imaging: How good are the data? J Am Coll Cardiol 2012;59:553-65.

10. Slomka PJ, Nishina H, Berman DS, Akincioglu C, Abidov A, Friedman JD, et al. Automated quantification of myocardial perfusion SPECT using simplified normal limits. J Nucl Cardiol 2005;12:66-77.

11. Klein R, Hung G, Wu T, Huang W-S, Li D, deKemp RA, et al. Feasibility and operator variability of myocardial blood flow and reserve measurements with $99 \mathrm{mTc}$-sestamibi quantitative dynamic SPECT/CT imaging. J Nucl Cardiol 2014. doi:10.1007/s12350014-9971-8.

12. Leppo JA, Meerdink DJ. Comparison of the myocardial uptake of a technetium-labeled isonitrile analogue and thallium. Circ Res 1989;65:632-9.

13. Yoshida K, Mullani N, Gould KL. Coronary flow and flow reserve by PET simplified for clinical applications using rubidium- 82 or nitrogen-13-ammonia. J Nucl Med 1996;37:1701-12.
14. Chua T, Kiat H, Germano G, Palmas W, Takemoto K, Friedman J, et al. Rapid back to back adenosine stress/rest technetium-99m teboroxime myocardial perfusion SPECT using a triple-detector camera. J Nucl Med 1993;34:1485-93.

15. Sherif HM, Nekolla SG, Saraste A, Reder S, Yu M, Robinson S, et al. Simplified quantification of myocardial flow reserve with flurpiridaz F 18: Validation with microspheres in a pig model. J Nucl Med 2011;52:617-24.

16. Alhassen F, Nguyen N, Bains S, Gould RG, Seo Y, Bacharach SL, et al. Myocardial blood flow measurement with a conventional dual-head SPECT/CT with spatiotemporal iterative reconstructions-A clinical feasibility study. Am J Nucl Med Mol Imaging 2013;4:53-9.

17. Slomka PJ, Alexanderson E, Jacome R, Jiménez M, Romero E, Meave A, et al. Comparison of clinical tools for measurements of regional stress and rest myocardial blood flow assessed with $13 \mathrm{~N}$ ammonia PET/CT. J Nucl Med 2012;53:171-81.

18. Dekemp RA, Declerck J, Klein R, Pan XB, Nakazato R, Tonge C, et al. Multisoftware reproducibility study of stress and rest myocardial blood flow assessed with 3D dynamic PET/CT and a 1-tissue-compartment model of $82 \mathrm{Rb}$ kinetics. J Nucl Med 2013;54:571-7.

19. Verger A, Imbert L, Yagdigul Y, Fay R, Djaballah W, Rouzet F, et al. Factors affecting the myocardial activity acquired during exercise SPECT with a high-sensitivity cardiac CZT camera as compared with conventional Anger camera. Eur J Nucl Med Mol Imaging 2014;41:522-8.

20. Slomka PJ, Dey D, Duvall WL, Henzlova MJ, Berman DS, Germano G. Advances in nuclear cardiac instrumentation with a view towards reduced radiation exposure. Curr Cardiol Rep 2012;14: 208-16.

21. Sharir T, Ben-Haim S, Merzon K, Prochorov V, Dickman D, BenHaim S, et al. High-speed myocardial perfusion imaging: Initial clinical comparison with conventional dual detector anger camera imaging. JACC Cardiovascular Imaging 2008;1:156-63.

22. Peretz A, Roth N. CZT scanners installed base (ASNC 2013 Personal communications). Chicago: ASNC; 2012.

23. Henzlova M, Duvall W. The future of SPECT MPI: Time and dose reduction. J Nucl Cardiol 2011;18:580-7.

24. Gimelli A, Bottai M, Genovesi D, Giorgetti A, Di Martino F, Marzullo P. High diagnostic accuracy of low-dose gated-SPECT with solid-state ultrafast detectors: Preliminary clinical results. Eur J Nucl Med Mol Imaging 2012;39:83-90.

25. Nakazato R, Berman D, Hayes M, Fish M, Padgett R, Xu Y, et al. Myocardial perfusion imaging with a solid state camera: Simulation of a very low dose imaging protocol. J Nucl Med 2013; 54:373-9.

26. Ben-Haim S, Murthy VL, Breault C, Allie R, Sitek A, Roth N, Fantony J, et al. Quantification of myocardial perfusion reserve using dynamic SPECT imaging in humans: A feasibility study. J Nucl Med 2013;54:873-9.

27. Wells RG, Timmins R, Klein R, Lockwood J, Marvin B, deKemp RA, et al. Dynamic SPECT measurement of absolute myocardial blood flow in a porcine model. J Nucl Med 2014;55:1685-91.

28. Imbert L, Poussier S, Franken PR, Songy B, Verger A, Morel O, et al. Compared performance of high-sensitivity cameras dedicated to myocardial perfusion SPECT: A comprehensive analysis of phantom and human images. J Nucl Med 2012;53:1897-903.

29. Mawlawi O, Podoloff DA, Kohlmyer S, Williams JJ, Stearns CW, Culp RF, et al. Performance characteristics of a newly developed PET/CT scanner using NEMA standards in $2 \mathrm{D}$ and $3 \mathrm{D}$ modes. J Nucl Med 2004;45:1734-42. 